\title{
Triagem virtual de inibidores da MAO-B, um alvo molecular na doença de Parkinson
}

\author{
Virtual screening for MAO-B inhibitors, a molecular target in \\ Parkinson's disease
}
Detección virtual de inhibidores de $M A O-B$, un objetivo molecular en la enfermedad de Parkinson

\author{
Joice Silva de Oliveira ${ }^{1}$, Luciana Fernandes Pastana Ramos² \\ 1.Farmacêutica, Graduação. Universidade Federal do Oeste do Pará, Santarém-PA, Brasil. \\ 2.Fisioterapeuta, Mestre em Neurociências, docente da Universidade Federal do Pará - UFPA, Belém-PA, \\ Brasil.
}

\begin{abstract}
Resumo
Introdução. A Doença de Parkinson (DP) é uma patologia neurológica caracterizada pela degeneração crônica e progressiva de neurônios dopaminérgicos na substância negra pars compacta (SNpc) e é a segunda desordem neurodegenerativa mais comum, sendo a idade o principal fator de risco. A L-3,4-dihidroxifenilanina (L-DOPA) tem sido amplamente utilizada como o principal tratamento de DP. Todavia, a maioria dos pacientes cronicamente tratados com L-DOPA apresentam efeitos adversos motores e psiquiátricos. Assim, várias estratégias terapêuticas têm sido testadas a fim de repor dopamina estriatal de maneira mais fisiológica, dentre elas a utilização de inibidores da Monoamina Oxidase B (MAO-B). Objetivo. Obter modelos teóricos de novos inibidores da MAO-B, com características físico-químicas para o desenvolvimento de fármacos para o tratamento da DP. Método. Foi realizada uma triagem virtual baseada na estrutura do inibidor cristalográfico Safinamida, com aplicação de filtro para avaliação da passagem pela barreira hematoencefálica, bem como simulações de docking e validação de re-docking. Resultados. Os inibidores teóricos foram: (7R,8S,8'R)-7-Hydroxy3,4,3',4'-tetramethoxy-8,8'-neolignan, Oxovirolin and 3-oxo-skimmiarepin, que exibiram afinidade para o sítio de ligação da MAO-B melhor ou semelhante ao do inibidor de referência. Todos os compostos exibiram propriedades físico-químicas favoráveis à biodisponibilidade oral, de acordo com os descritores de Lipinski e Veber. Conclusão. Os inibidores teóricos encontrados são alvos potenciais para o planejamento e desenvolvimento de medicamentos para a doença de Parkinson. Porém, por se tratar de um estudo in silico, é necessário que mais pesquisas e testes sejam feitos para avaliar in vitro e, posteriormente, in vivo o comportamento dessas moléculas.
\end{abstract}

Unitermos. Inibidores enzimáticos; Neuroproteção; Terapia medicamentosa; Produtos Biológicos

\footnotetext{
Abstract

Introduction. Parkinson's disease (PD) is a neurological pathology characterized by chronic and progressive degeneration of dopaminergic neurons in the substantia nigra pars compact (SNpc) and is the second most common neurodegenerative disorder, with age being the main risk factor. L-3,4-dihydroxyphenylanine (L-DOPA) has been widely used as the main treatment for PD. However, the majority of patients chronically treated with L-DOPA have adverse motor and psychiatric effects. Thus, several therapeutic strategies have been tested in order to replace striatal dopamine in a more physiological way, among them the use of Monoamine Oxidase B (MAO-B) inhibitors. Objective. To obtain theoretical models of new MAO-B inhibitors, with physico-chemical characteristics for the development of drugs for the treatment of PD. Method. A virtual screening was performed based on the structure of the crystallographic inhibitor Safinamide, with filter application to evaluate the passage through the blood-brain barrier, as well as docking simulations and re-docking validation. Results. The theoretical inhibitors were: (7R,8S,8'R)-7-Hydroxy-3,4,3',4'-tetramethoxy-8,8'-neolignan,
} 
Oxovirolin and 3-oxo-skimmiarepin, which exhibited affinity to the MAO-B binding site better or similar to that of the reference inhibitor. All compounds exhibited physicochemical properties favorable for oral bioavailability, according to the descriptors of Lipinski and Veber. Conclusion. The theoretical inhibitors found are potential targets for planning and designing drugs for Parkinson's Disease. However, because it is an in silico study, it is necessary that more research and tests are done to evaluate in vitro and, later, in vivo the behavior of these molecules.

Keywords. Enzyme Inhibitors; Neuroprotection; Drug Therapy; Biological Products

\section{Resumen}

Introducción. La enfermedad de Parkinson (EP) es una patología neurológica caracterizada por la degeneración crónica y progresiva de las neuronas dopaminérgicas en la sustancia negra pars compacta (SNpc) y es el segundo trastorno neurodegenerativo más común, siendo la edad el principal factor de riesgo. La L-3,4-dihidroxifenilanina (L-DOPA) se ha utilizado ampliamente como tratamiento principal para la EP. Sin embargo, la mayoría de los pacientes tratados crónicamente con L-DOPA tienen efectos motores y psiquiátricos adversos. Así, se han ensayado varias estrategias terapéuticas con el fin de sustituir la dopamina estriatal de una forma más fisiológica, entre ellas el uso de inhibidores de la monoamino oxidasa B (MAOB). Objetivo. Obtener modelos teóricos de nuevos inhibidores de la MAO-B, con características físico-químicas para el desarrollo de fármacos para el tratamiento de la EP. Método. Se realizó un cribado virtual basado en la estructura del inhibidor cristalográfico de Safinamida, con la aplicación de un filtro para evaluar el paso a través de la barrera hematoencefálica, así como simulaciones de atraque y validación de reenganche. Resultados. Los inhibidores teóricos fueron: (7R, 8S, 8'R) -7-Hidroxi-3,4,3 ', 4'-tetrametoxi-8,8'-neolignan, Oxovirolina y 3-oxoskimmiarepin, que mostraron afinidad por el sitio de unión de MAO-B mejor o similar al del inhibidor de referencia. Todos los compuestos exhibieron propiedades fisicoquímicas favorables a la biodisponibilidad oral, según los descriptores de Lipinski y Veber. Conclusiones. Los inhibidores teóricos encontrados son objetivos potenciales para la planificación y el desarrollo de fármacos para la enfermedad de Parkinson. Sin embargo, por tratarse de un estudio in silico, es necesario que se realicen más investigaciones y pruebas para evaluar in vitro $y$, posteriormente, in vivo el comportamiento de estas moléculas.

Palabras clave. Inhibidores de enzimas; Neuroprotección; Terapia de drogas; Productos biológicos

Trabalho realizado na Universidade Federal do Oeste do Pará, Santarém-PA, Brasil.

Endereço para correspondência: Joice Silva de Oliveira. R. Elísio de Carvalho 90, Apto 7. Vila São Luis. São Paulo-SP, Brasil. CEP 05362-070. Email: joicesdoliveira@gmail.com

\section{INTRODUÇÃO}

A Doença de Parkinson (DP) é uma patologia neurológica caracterizada pela degeneração crônica e progressiva de neurônios dopaminérgicos na substância negra pars compacta (SNpc), redução dos níveis de dopamina na via nigroestriatal e desenvolvimento de agregados proteicos citoplasmáticos intraneurais, conhecidos como corpos de Lewy ${ }^{1-3}$. Dados epidemiológicos 
revelam que a DP é a segunda desordem neurodegenerativa mais comum. A idade é o principal fator de risco e os pacientes mais idosos geralmente apresentam alterações clínicas mais severas. A prevalência estimada em países industrializados é de $0,3 \%$ na população geral, $1 \%$ em pessoas acima de 60 anos e $3 \%$ naqueles com 80 anos ou mais. A taxa de incidência é de $8-18 / 100$ mil pessoas por $\mathrm{ano}^{4}$.

Apesar da primeira descrição da patologia ter sido publicada em 1817, até hoje não se conhece a etiologia da DP. O diagnóstico clínico é realizado através da detecção dos quatro sinais cardinais motores de parkinsonismo: bradicinesia, tremor em repouso, rigidez muscular e instabilidade postural ${ }^{1}$. No entanto, a DP também inclui sintomas não motores, tais como: distúrbios de humor e afeto, distúrbios do sono, comprometimento cognitivo (incluindo disfunção executiva frontal e alucinações), disautonomia (hipotensão ortostática, disfunção urogenital e constipação), além de sintomas sensoriais (hiposmia) e $\operatorname{dor}^{5,6}$.

Outra forma de diagnosticar o parkinsonismo é através da administração oral de Levodopa seguida de ausência ou diminuição dos sintomas motores. A L-3,4dihidroxifenilanina (L-DOPA), um precursor de dopamina, foi descrita em 1962 como terapia efetiva em reduzir os déficits motores presente no parkinsonismo e, ainda hoje, tem sido amplamente utilizada como o principal tratamento de DP7. Todavia, a maioria dos pacientes cronicamente tratados com 
L-DOPA apresentam efeitos adversos motores e psiquiátricos, tais como a discinesia e alucinações. Assim, desde a década de 70, várias estratégias terapêuticas têm sido testadas a fim de repor dopamina estriatal de maneira mais fisiológica ${ }^{6,7}$.

Outra estratégia terapêutica prescrita aos parkinsonianos é a utilização de inibidores da Monoamina Oxidase B (MAO-B) ${ }^{1}$. Estes, além de potencializar as ações da dopamina, apresentam um efeito benéfico nas flutuações motoras, sugerindo melhor controle sintomático a longo prazo com a terapia prévia a Levodopa do que com a Levodopa em $\mathrm{si}^{5,8}$. Foi demonstrado ainda que a inibição da MAO-B produz um efeito neuroprotetor por meio de mecanismos anti-apoptóticos, atenuação da produção de espécies reativas de oxigênio (ERO) e estabilização da membrana mitocondrial ${ }^{9,10}$.

Somado a isto, é comprovado que a atividade enzimática da monoamina oxidase $B$ no encéfalo aumenta com a idade e, geralmente, é maior que o normal em pacientes afetados por doenças neurodegenerativas apresentando uma correlação direta com 0 comprometimento cognitivo e a neurodegeneração causada pelo estresse oxidativo ${ }^{9}$.

Derivados da propargilamina revelaram atividades inibitórias seletivas e irreversíveis da MAO-B, entre eles: Selegilina e Rasagilina. Ambos são usados como monoterapia no início do tratamento e terapia adjunta com os agonistas da L-DOPA e dopamina nos estágios posteriores 
da $\mathrm{DP}^{8,10}$. A Safinamida, um inibidor reversível, também foi desenvolvido para este propósito ${ }^{10}$.

O tratamento crônico com os inibidores seletivos da MAO-B melhora a liberação de DA, provavelmente devido à elevação das aminas cerebrais endógenas ou a modulação do receptor alvo. Contudo, inibidores não seletivos da MAO induzem aumentos significativos nos níveis de DA no estriado e em outras regiões ${ }^{11}$.

A Selegilina e a Rasagilina são submetidas a um extenso metabolismo hepático ${ }^{8}$. A Selegilina produz metabólitos de anfetamina que contribuem para a inibição da recaptação de norepinefrina, potencializando os efeitos farmacológicos da L-DOPA. Contudo, esses metabólitos têm sido associados a efeitos colaterais cardiovasculares (hipotensão ortostática) e psiquiátricos (alucinações), intensificando os sintomas não motores ${ }^{8}$.

Rasagilina, além da atividade inibidora da MAO-B, apresentou favoráveis atividades antioxidantes e antiapoptóticas in vitro e levou à indução de fatores neurotróficos ${ }^{8}$. A Safinamida é um inibidor seletivo reversível da MAO-B derivado da a-aminoamida indicado para o tratamento da DP inicial. Atua também como modulador do glutamato, além de possuir atividade bloqueadora de canais de sódio e cálcio dependentes de voltagem ${ }^{3,8}$.

Recentemente, tem sido relatado que uma série de furanochalconas exibiram atividades inibitórias moderadas a boas em relação à MAO-B, com o composto mais ativo, 2E3-(5-clorofuran-2-il)-1-(3-clorofenil)prop-2-en-1-ona, 
exibindo um valor de IC50 de 0,174 $\mu$ M para a forma humana da enzima ${ }^{10,12}$. Em um trabalho subsequente, derivados heterocíclicos de chalconas foram examinados como inibidores das MAOs, cujo inibidor mais potente também apresentou especificidade para MAO-B ${ }^{10,13}$.

Considerando que a inibição da MAO-B é um alvo biológico promissor no tratamento da Doença de Parkinson, o presente trabalho teve como objetivo a obtenção de modelos teóricos de inibidores de MAO-B que possuam características físico-químicas adequadas ao desenvolvimento de drogas para o Sistema Nervoso Central, com potencial capacidade de alta permeabilidade à barreira hematoencefálica e alta afinidade e seletividade ao receptor. Para tal, foram utilizados métodos in silico de Química Medicinal com abordagens computacionais voltadas para 0 planejamento racional de fármacos.

\section{MÉTODO}

\section{Obtenção da estrutura cristalográfica 3D do alvo} molecular

A estrutura cristalográfica da enzima alvo, Monoamina Oxidase B (MAO-B), encontra-se disponível no banco de dados de proteínas PDB (Protein Data Bank), sob o código de acesso $\mathbf{2} \mathbf{V 5 Z} \mathbf{Z}^{14}$. Considerou-se a resolução $(R)$ da estrutura experimental $(R=1,60 \AA)$ e a presença de um inibidor cristalográfico, Safinamida (SAG), para a comparação com as moléculas em estudo. 


\section{Seleção do banco de moléculas}

Foi selecionado para estudo o banco de moléculas do NuBBE (Núcleo de Bioensaios, Biossíntese e Ecofisiologia de Produtos Naturais) da Universidade Estadual Paulista (UNESP) e do Laboratório de Química Medicinal e Computacional (LQMC) da Universidade de São Paulo (USP). Trata-se de uma base de dados que abrange uma variedade de produtos naturais isolados em NuBBE e seus derivados, bem como as propriedades químicas e farmacológicas úteis desses compostos.

\section{Definição do modelo farmacofórico do inibidor}

Para a definição do modelo farmacofórico da Safinamida, utilizou-se o servidor Pharmit. Primeiro, deve ser inserido o código de acesso da proteína no PDB (2V5Z) e selecionado o ligante desejado (SAG, Safinamida). Como os banco de dados do NuBBE disponíveis no Pharmit eram incompletos, submetemos nosso próprio banco de dados NuBBE, com 2.149 confôrmeros de 2.149 moléculas disponíveis.

Foram definidos três modelos farmacofóricos, tomando como base dados da literatura sobre as interações intermoleculares: modelo 1: quatro ligações hidrofóbicas; modelo 2: quatro ligações hidrofóbicas e uma ligação de hidrogênio ( $O$ da amida como aceptor); modelo 3: quatro ligações hidrofóbicas e uma ligação de hidrogênio ( $N$ como aceptor), conforme exibido na Figura 1. 
Figura 1. Modelos farmacofóricos do inibidor cristalográfico. (A) Modelo 1: 4 ligações hidrofóbicas (verde), (B) Modelo 2: 4 ligações hidrofóbicas (verde) e 1 ligação de hidrogênio (amarelo) e (C) Modelo 3: 4 ligações hidrofóbicas (verde) e 1 ligação de hidrogênio(amarelo).

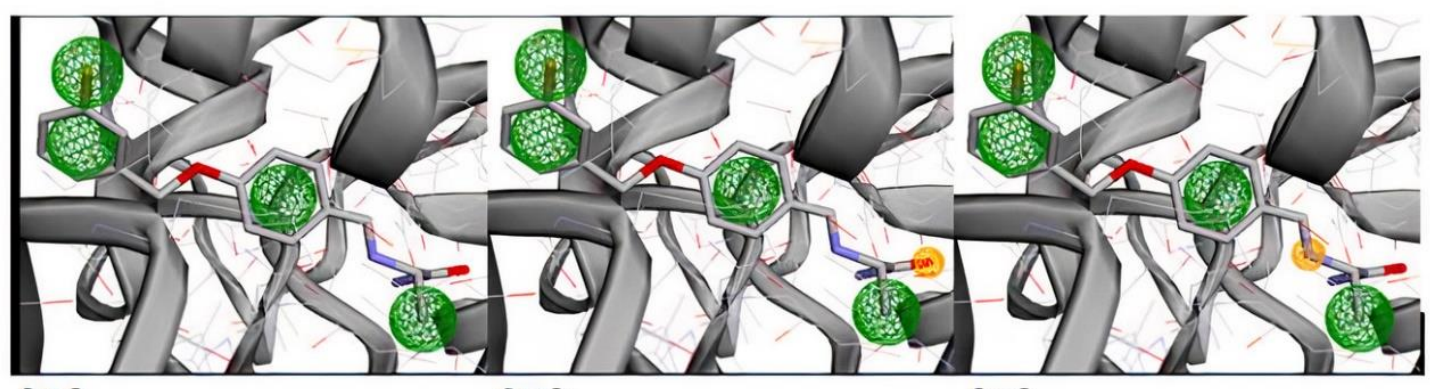

(A)

(B)

(C)

\section{Filtragem de moléculas}

A filtragem das mais de duas mil moléculas do NuBBE foi feita utilizando como base os três modelos farmacofóricos determinados no Pharmit. Antes da realização da filtragem, fez-se a remoção dos hits duplicados do banco de dados através do software livre Osiris Data Warrior ${ }^{15}$.

\section{Avaliação da penetração na barreira hematoencefálica}

Uma vez que o alvo molecular é uma enzima localizada na região do encéfalo, é fundamental que o composto candidato à fármaco seja capaz de atravessar a barreira hematoencefálica. Portanto, foi realizada nova filtragem de moléculas por meio do filtro CNS (Central Nervous System), disponível no servidor FAFDrugs4.

\section{Protocolo de Docking Molecular}

O protocolo de docking ou atracamento molecular foi executado através do programa Molegro Virtual Docker 
(MVD) ou MolDock. O MolDock é um programa baseado em um algoritmo de busca heurístico, denominado evolução diferencial combinado com a predição de cavidades $^{16}$.

O MVD disponibiliza um módulo de preparação automatizado para docking, capaz de corrigir os tipos de átomos e as ordens de ligação para as estruturas dos ligantes e das proteínas, além de adicionar átomos de hidrogênio para cada complexo e definir cargas atômicas parciais. Durante a execução do atracamento molecular, moléculas de água, cofatores e íons foram removidos.

Como o PDB não possui a estrutura completa da enzima alvo (520 aminoácidos em cada cadeia): no monômero $A$ até Thr-500; no monômero $B$ até Ile-496, e por se tratar de uma proteína homodímera (duas cadeias idênticas), escolhemos a cadeia A para protocolo de docking por apresentar uma cadeia mais completa que a $\mathrm{B}^{17}$. Foi realizado atracamento molecular para as moléculas aceitas no filtro CNS e, ao final, utilizou-se os valores de energia (Rerank score) como base para a seleção de três melhores poses de docking. Os valores de energia são definidos em $\mathrm{kcal} / \mathrm{mol}$ e devem ser inferiores a zero.

\section{Análise de interações}

Os três compostos que exibiram melhores valores de energia após o docking e o inibidor cristalográfico sofreram análise de interações realizada no servidor online PLIP (Protein-Ligand Interaction Profiler), capaz de identificar interações intermoleculares (não-covalentes) entre 
proteínas e ligantes de maneira fácil e rápida. Na página de resultados, o PLIP lista todos os dados de interação, agrupados por tipo de ligante (moléculas pequenas, DNA/RNA, íons, polímeros) e, eventualmente, complexos simples, além de disponibilizar os detalhes de cada interação em tabelas.

\section{Estudo das propriedades físico-químicas dos compostos naturais}

Para o estudo dos compostos, foi utilizado um banco de dados de química aberto no National Institutes of Health $(N I H)$, o PubChem ${ }^{18-20}$. Este banco de dados contém informações sobre estruturas químicas, identificadores, propriedades químicas e físicas, atividades biológicas, patentes, saúde, segurança, dados de toxicidade e outros.

\section{Validação dos Protocolos de Docking por Re-docking}

$\mathrm{Na}$ validação por re-docking, o processo de docking molecular é realizado com o próprio ligante da estrutura cristalina (Safinamida) de um complexo ligante-proteína a fim de tentar reproduzir o modelo de ligação original. A enzima (MAO-B) foi considerada como um corpo rígido e suas moléculas de água originais foram removidas ${ }^{16}$.

A credibilidade dos resultados de docking depende da similaridade entre o modo de ligação experimental e a pose de menor energia $(\mathrm{kcal} / \mathrm{mol})$. Para tal análise, é considerado o valor de RMSD (Root-Mean-Square Deviation, ou seja, Desvio Quadrático Médio das Distâncias) em angstroms ( $\AA$ ). Um valor muito alto de RMSD significa que os compostos em 
comparação são diferentes, e zero significa que eles são idênticos em conformação ${ }^{21}$. Neste trabalho, foi considerado aceitável como metodologia validada, todo resultado que apresentou valor de RMSD inferior ou equivalente a 1,0.

\section{RESULTADOS}

\section{Filtragem de moléculas}

$\mathrm{Na}$ filtragem pelos três modelos farmacofóricos determinados no Pharmit, foram obtidos 382 hits no banco de moléculas do NuBBE. Entretanto, após a remoção dos hits duplicados, restaram somente 174 .

\section{Avaliação da penetração na barreira hematoencefálica}

Dos 174 hits restantes da primeira filtragem, somente 9 moléculas foram aceitas pelo filtro CNS (Tabela 1), indicando que somente aproximadamente $5,2 \%$ das moléculas possuem as características físico-químicas necessárias para ultrapassar a barreira hematoencefálica.

\section{Protocolo de Docking Molecular}

O atracamento molecular foi executado para as 9 moléculas aprovadas no filtro CNS, juntamente com o inibidor Safinamida na cadeia A da enzima MAO-B. De acordo com o Rerank score, as três melhores poses de docking foram: (1) (7R, 8S, 8'R)-7-Hydroxy-3,4,3',4'-tetramethoxy8,8'-neolignan, com valor de energia igual a $124,266 \mathrm{kcal} / \mathrm{mol}$; (2) Oxovirolin, com energia de $123,797 \mathrm{kcal} / \mathrm{mol}$; e (3) 3-oxo-skimmiarepin, apresentando 
energia de $-113,261 \mathrm{kcal} / \mathrm{mol}$. A Safinamida (SAG 1503 [A]) apresentou energia de ligação $-114,202 \mathrm{kcal} / \mathrm{mol}$. O protocolo de docking molecular revelou que duas das três moléculas em questão exibiram valores de energia melhores que a do inibidor cristalográfico, sendo a Safinamida superior apenas em relação ao 3-oxo-skimmiarepin.

Tabela 1. Moléculas aceitas pelo filtro CNS (Fonte: NuBBE).

\begin{tabular}{lcc}
\hline NuBBE ID & Nome Vulgar & Classe \\
\hline NuBBE 381 & 22-hidroxi-tingenona; tingenina B & Terpenos: triterpeno \\
\hline NuBBE 1005 & 8,14-epoxyergosta-4,22-diene-3,6-dione & Terpenos: esteroides \\
\hline NuBBE 1146 & Piscidinol A & Terpenos: triterpeno \\
\hline NuBBE 1406 & 21a,25-Dimetilmelianodiol & Terpenos: triterpeno \\
\hline NuBBE 1523 & Melianona & Terpenos: triterpeno \\
\hline NuBBE 1519 & 213,25-Dimetilmelianodiol & Terpenos: triterpeno \\
\hline NuBBE 1583 & 3-oxo-skimmiarepin & $\begin{array}{c}\text { Terpenos: } \\
\text { triterpenoides }\end{array}$ \\
\hline NuBBE 1668 & $\begin{array}{c}\text { (7R,8S,8'R)-7-Hydroxy-3,4,3',4'- } \\
\text { tetramethoxy-8,8'-neolignan }\end{array}$ & Lignóides \\
\hline NuBBE 2435 & Oxovirolin; & Lignóides \\
\hline
\end{tabular}

\section{Validação dos Protocolos de Docking por Re-docking}

Para o re-docking utilizou-se a estrutura do próprio ligante cristalográfico Safinamida, o qual passou por um novo protocolo de docagem molecular, visando reproduzir o modelo de ligação original. A comparação por sobreposição de estruturas do complexo cristalográfico com a melhor pose de docking da Safinamida revelou um modo de ligação 
similar à conformação original com RMSD de $0,76 \AA$ (Figura 2), estando dentro do valor aceitável como válido (até $1,0 \AA$ ) e validando positivamente o protocolo de docking.

Figura 2. Sobreposição do ligante Safinamida obtido por cristalografia (cinza) e da melhor pose de docking (azul) com RMSD igual a 0,76 .

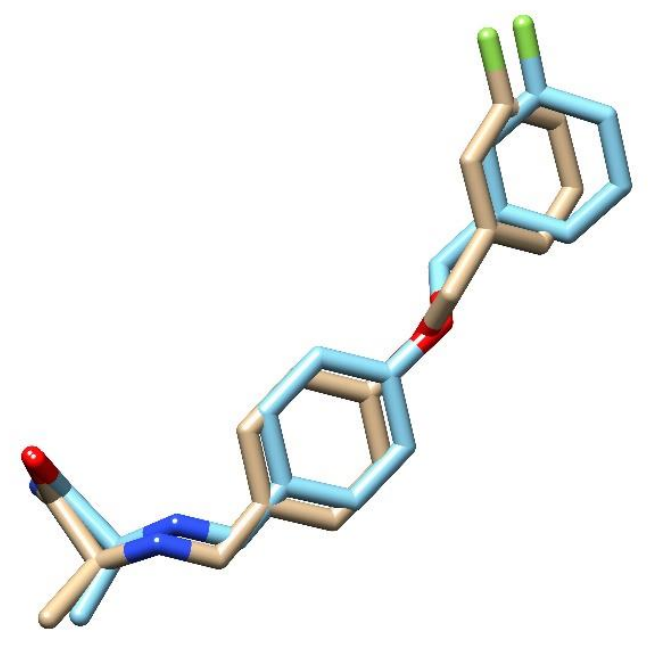

\section{Análise de interações}

O complexo ligante-proteína do (7R,8S,8'R)-7Hydroxy-3,4,3', 4'-tetramethoxy-8,8'-neolignan foi capaz de realizar dez interações hidrofóbicas e duas ligações de hidrogênio. Nas interações hidrofóbicas participaram os resíduos Phe-168, Leu-171, Ile-198, Ile-199, Tyr-326, Tyr398 e Tyr-435, sendo que três destes (Leu-171, Ile-199 e Tyr-398) interagiram mais de uma vez. As ligações de hidrogênio ocorreram entre o receptor e os resíduos Tyr-60 e Leu-171. Com a tirosina (Tyr), o ligante apresentou-se como aceptor de hidrogênio e o resíduo como doador, enquanto com a leucina (Leu) foi o oposto. Todas as 
interações deste complexo apresentaram distância de ligação igual ou inferior à 4,0 $\AA$ (Tabela 2 ) e são exibidas na Figura 3.

Tabela 2. Interações intermoleculares entre os átomos das cadeias laterais dos resíduos e dos ligantes.

\begin{tabular}{|c|c|c|c|c|c|c|c|}
\hline \multicolumn{4}{|c|}{$\begin{array}{c}\text { (7R,8S,8'R)-7-Hydroxy-3,4,3',4'- } \\
\text { tetramethoxy-8,8'-neolignan }\end{array}$} & \multicolumn{4}{|c|}{ 3-oxo-skimmiarepin } \\
\hline \multicolumn{4}{|c|}{ Interacões Hidrofóbicas } & \multicolumn{4}{|c|}{ Interacões Hidrofóbicas } \\
\hline Resíduo & AA & \multicolumn{2}{|c|}{ Distância $(\hat{A})$} & Resíduo & AA & \multicolumn{2}{|c|}{ Distância $(\hat{A})$} \\
\hline $168 \mathrm{~A}$ & PHE & \multicolumn{2}{|c|}{3,12} & $171 \mathrm{~A}$ & LEU & \multicolumn{2}{|c|}{3,02} \\
\hline $171 \mathrm{~A}$ & LEU & \multicolumn{2}{|c|}{3,35} & $199 \mathrm{~A}$ & ILE & \multicolumn{2}{|c|}{3,31} \\
\hline $171 \mathrm{~A}$ & LEU & \multicolumn{2}{|c|}{3,29} & $206 \mathrm{~A}$ & GLN & \multicolumn{2}{|c|}{3,63} \\
\hline $198 \mathrm{~A}$ & ILE & \multicolumn{2}{|c|}{3,59} & $326 \mathrm{~A}$ & TYR & \multicolumn{2}{|c|}{3,62} \\
\hline $199 \mathrm{~A}$ & ILE & \multicolumn{2}{|c|}{3,93} & $326 \mathrm{~A}$ & TYR & \multicolumn{2}{|c|}{3,16} \\
\hline $199 \mathrm{~A}$ & ILE & \multirow{2}{*}{\multicolumn{2}{|c|}{$\begin{array}{l}3,81 \\
3,87\end{array}$}} & $343 \mathrm{~A}$ & PHE & \multirow{2}{*}{\multicolumn{2}{|c|}{3,71}} \\
\hline $326 \mathrm{~A}$ & TYR & & & $398 \mathrm{~A}$ & TYR & & \\
\hline $398 \mathrm{~A}$ & TYR & \multicolumn{2}{|c|}{3,37} & $398 \mathrm{~A}$ & TYR & \multicolumn{2}{|c|}{3,76} \\
\hline $398 \mathrm{~A}$ & TYR & \multicolumn{2}{|c|}{3,60} & $435 \mathrm{~A}$ & TYR & \multicolumn{2}{|c|}{$\begin{array}{l}3,63 \\
3,02\end{array}$} \\
\hline $435 \mathrm{~A}$ & TYR & \multirow{2}{*}{\multicolumn{2}{|c|}{3,42}} & $435 \mathrm{~A}$ & TYR & \multirow{2}{*}{\multicolumn{2}{|c|}{$\begin{array}{l}3,69 \\
3,28\end{array}$}} \\
\hline & & & & $435 \mathrm{~A}$ & TYR & & \\
\hline \multicolumn{4}{|c|}{ Ligações de Hidrogênio } & & gaçõe & Hidrogênic & \\
\hline Resíduo & AA & $\begin{array}{c}\text { Distância } \\
\text { AH (Â) }\end{array}$ & $\begin{array}{c}\text { Distância } \\
\text { DH }(\hat{A})\end{array}$ & Resíduo & AA & $\begin{array}{c}\text { Distância } \\
\text { AH }(\hat{A})\end{array}$ & $\begin{array}{c}\text { Distância } \\
\mathrm{DH}(\hat{\mathrm{A}})\end{array}$ \\
\hline $60 \mathrm{~A}$ & TYR & 3,09 & 4,00 & $198 \mathrm{~A}$ & ILE & 2,18 & 2,67 \\
\hline $171 \mathrm{~A}$ & LEU & 1,84 & 2,87 & $398 \mathrm{~A}$ & TYR & 2,54 & 3,10 \\
\hline & & & & $434 \mathrm{~A}$ & GLY & 2,26 & 3,25 \\
\hline & & irolin & & Safina & (In & r de crist & grafia) \\
\hline & terac & Hidrofóbica & & & terac & iidrofóbica & \\
\hline Resíduo & AA & Dist & $\mathrm{a}(\hat{\mathrm{A}})$ & Resíduo & $\mathrm{AA}$ & Distâ & $\mathrm{a}(\hat{\mathrm{A}})$ \\
\hline $60 \mathrm{~A}$ & TYR & & & $168 \mathrm{~A}$ & $\mathrm{PHE}$ & & \\
\hline $168 \mathrm{~A}$ & PHE & & & $171 \mathrm{~A}$ & LEU & & \\
\hline $171 \mathrm{~A}$ & LEU & & & $171 \mathrm{~A}$ & LEU & & \\
\hline $171 \mathrm{~A}$ & LEU & & & $199 \mathrm{~A}$ & ILE & & \\
\hline $199 \mathrm{~A}$ & ILE & & & $398 \mathrm{~A}$ & TYR & & \\
\hline $199 \mathrm{~A}$ & ILE & & & $398 \mathrm{~A}$ & TYR & & \\
\hline $326 \mathrm{~A}$ & TYR & & & & & & \\
\hline $343 \mathrm{~A}$ & PHE & & & & & & \\
\hline $343 \mathrm{~A}$ & $\mathrm{PHE}$ & & & & & & \\
\hline & giaçõ & Hidrogêni & & & gaçõe & Hidrogênic & \\
\hline Resíduo & AA & $\begin{array}{c}\text { Distância } \\
\mathrm{AH}(\hat{\mathrm{A}})\end{array}$ & $\begin{array}{l}\text { Distância } \\
\mathrm{DH}(\hat{\mathrm{A}})\end{array}$ & Resíduo & AA & $\begin{array}{c}\text { Distância } \\
\mathrm{AH}(\hat{\mathrm{A}})\end{array}$ & $\begin{array}{c}\text { Distância } \\
\text { DH }(\hat{A})\end{array}$ \\
\hline $198 \mathrm{~A}$ & ILE & 2,25 & 3,21 & $59 \mathrm{~A}$ & SER & 2,24 & 3,10 \\
\hline & & & & $60 \mathrm{~A}$ & TYR & 2,59 & 3,22 \\
\hline & & & & $60 \mathrm{~A}$ & TYR & 2,28 & 3,20 \\
\hline & & & & $60 \mathrm{~A}$ & TYR & 1,62 & 2,60 \\
\hline & & & & $436 \mathrm{~A}$ & MET & 2,82 & 3,81 \\
\hline & ções & npilhamen & & & cões & ipilhament & \\
\hline Resíduo & AA & Dist & $\mathrm{a}(\hat{\mathrm{A}})$ & Resíduo & $\mathrm{AA}$ & Distâ & $\mathrm{a}(\hat{\mathrm{A}})$ \\
\hline $398 \mathrm{~A}$ & TYR & & & $436 \mathrm{~A}$ & TYR & & \\
\hline
\end{tabular}

Para o oxovirolin, ocorreram nove interações hidrofóbicas com o receptor, sendo uma com cada resíduo a seguir: Tyr-60, Phe-168, Tyr-326, e duas interações com a 
Leu-171, a Ile-199 e a Phe-343. Este composto ainda interagiu por meio de uma ligação de hidrogênio com a Ile198, na qual o resíduo comportou-se como aceptor de hidrogênio, e uma ligação de empilhamento n (com a Tyr398). Com exceção da ligação n que apresentou distância de $4,76 \AA$, as demais interações exibiram distância igual ou inferior à 3,69 $\AA$ (Tabela 2).

Figura 3. Estrutura química dos compostos (1) e Interações com o sítio ativo da enzima (2), para (A) (7R,8S,8'R)-7-Hydroxy-3,4,3',4'-tetramethoxy-8, 8'-neolignan, (B) Oxovirolin, (C) 3-oxo-skimmiarepin e (D) Safinamida. Moléculas em azul representam os resíduos proteicos; em laranja os ligantes. Interações hidrofóbicas são representadas por traços pontilhados, as ligações de hidrogênio por uma linha reta azul e o empilhamento $n$ por uma linha tracejada verde.

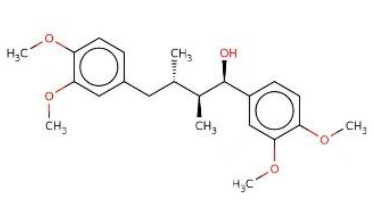

(A1)

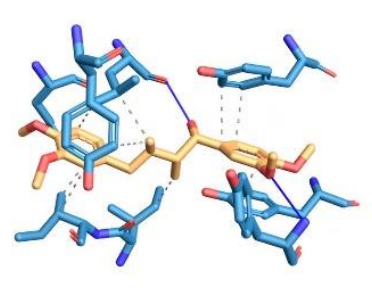

(A2)

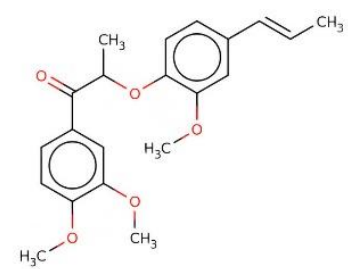

(B1)

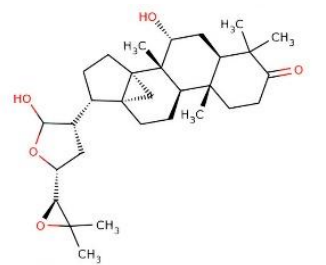

(C1)

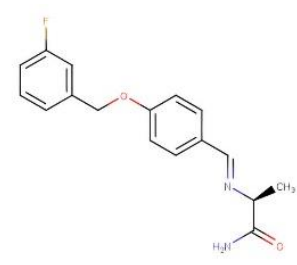

(D1)

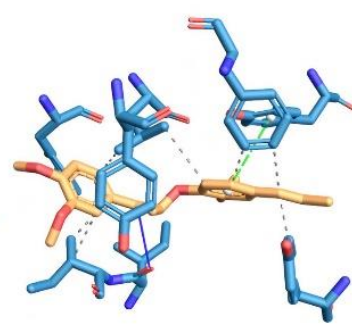

(B2)

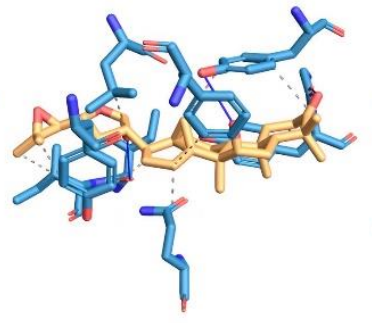

(C2)

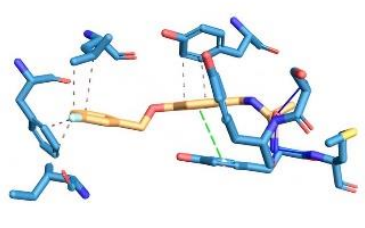

(D2)

O composto 3-oxo-skimmiarepin foi o que mais exibiu interações hidrofóbicas, sendo onze ao total: os resíduos Leu-171, Ile-199, GIn-206, Tyr-326, Phe-343, Tyr-398 e Tyr-435 interagiram uma única vez com o ligante; Tyr-326 e Tyr-398 fizeram duas interações; e Tyr-453 exibiu três 
diferentes interações. Este também foi, dos três, o que exibiu maior número de interações de hidrogênio, sendo três ao total, com os resíduos Ile-198, Tyr-398 e Gly-434 e, em todos os casos, os resíduos atuaram como aceptores de hidrogênio. Todas as interações exibiram distância igual ou inferior à 3,76 ̊, conforme Tabela 2 .

Pela análise dos resultados observa-se que os três compostos em estudo fizeram mais interações hidrofóbicas com as cadeias laterais dos resíduos do que a Safinamida, incluindo o 3-oxo-skimmiarepin (Tabela 2 ). Em relação às ligações de hidrogênio, a Safinamida foi a mais capaz de realizar essas interações com a cavidade da enzima e, além destas, somente ele e o oxovirolin exibiram interações de empilhamento $п$ (ou $n$-stacking).

\section{Estudo das propriedades físico-químicas dos compostos naturais}

O composto

(7R,8S, 8'R)-7-Hydroxy-3,4,3',4'tetramethoxy-8,8'-neolignan (nome comum), possui nome IUPAC (1R,2S,3S)-1,4-bis(3,4-dimethoxyphenyl)-2,3dimethylbutan-1-ol e fórmula molecular $\mathrm{C}_{22} \mathrm{H}_{30} \mathrm{O}_{5}$. Foi isolado da planta Lauraceae Nectandra puberula, em Cananeia-SP e pertence à classe dos Lignóides. Seu ID no banco de dados do NuBBE é $1668^{18}$.

O oxovirolin (NuBBE ID 2435) apresenta nome IUPAC 1-(3,4-dimethoxyphenyl)-2-\{2-methoxy-4-[(1E)-prop-1en-1-yl]phenoxy\}propan-1-one, e fórmula molecular $\mathrm{C}_{21} \mathrm{H}_{24} \mathrm{O}_{5}$, de fonte semissintética obtido da espécie 
Myristicaceae Virola surinamensis, em Belém-PA e também é pertencente à classe dos Lignóides ${ }^{19}$.

Oxovirolin já foi testado biologicamente contra leucemia e para análise de atividade antifúngica e atividade antileishmanial. Para o rastreio de drogas anticancerígenas, o oxovirolin apresentou-se inativo, bem como para os testes de atividade antifúngica contra: Candida albicans; Saccharomyces cerevisiae; Cryptococcus neoformans; Aspergillus niger; Aspergillus fumigatus; e Aspergillus flavus ${ }^{19}$.

Para o teste de toxicidade contra macrófagos de camundongos BALB/C avaliados como viabilidade celular e atividade antileishmanial contra Leishmania donovani e Leishmania amazonenses, o oxovirolin apresentou resultado de bioatividade não especificada. Também foi inespecífico para o teste de atividade antifúngica contra: Microsporum canis; Microsporum gypseum; Trichophyton mentagrophytes; Trichophyton rubrum e Epidermophyton floccosum ${ }^{19}$.

O composto 3-oxo-skimmiarepin apresenta NuBBE ID 1583 e nome IUPAC (1S,2R,3R,5R,10R,11R,14R,15S)-15[(3S,5R)-5-[(2S)-3,3-dimethyloxiran-2-yl]-2-hydroxyoxolan-3-yl]-3-hydroxy-2,6,6,10tetramethylpentacyclo $\left[12.3 .1 .0^{1}, 14.0^{2}, 11.0^{5}, 10\right]$ octadecan 7-one. Foi isolado da espécie Zanthoxylum petiolare, em Luis Antonio-SP, com fórmula molecular $\mathrm{C}_{30} \mathrm{H}_{46} \mathrm{O}_{5}{ }^{20}$. É um terpeno, da classe dos triterpenos. 
Foram analisadas as propriedades relacionadas à Regra dos Cinco de Lipinski²2, a qual prediz que para uma droga ter boa biodisponibilidade oral deve apresentar: peso molecular (PM) de até $500 \mathrm{Da}$; lipofilicidade (LogP) entre -1 e 5; até 10 aceptores de hidrogênio (HBA); e até 5 doadores de hidrogênio (HBD). Além dos descritores de Lipinski, analisamos também quanto a área de superfície polar (TPSA) e número de ligações rotacionáveis (NRB). De acordo com o descrito por $\operatorname{Veber}^{23}$, a TPSA de até $140 \AA$ e o NRB entre 1 e 10 indicam propriedades favoráveis a uma boa biodisponibilidade oral de fármacos. Os resultados são exibidos na Tabela 3.

Tabela 3. Propriedades físico-químicas dos ligantes, de acordo com os parâmetros analisados por Lipinski e Veber, respectivamente.

PARÂMETROS FÍSICO-QUÍMICOS

\begin{tabular}{|c|c|c|c|c|c|c|}
\hline LIGANTE & $\mathrm{PM}^{\mathrm{a}}(\mathrm{g} / \mathrm{mol})$ & LogP b & $\mathrm{HBA}^{\mathrm{c}}$ & $\mathrm{HBD}^{\mathrm{d}}$ & $\operatorname{TPSA}^{\mathrm{e}}(\AA)$ & $\mathrm{NRB}^{\mathrm{f}}$ \\
\hline NuBBE $1668^{1}$ & 374,47 & 3,72 & 5 & 1 & 57,16 & 9 \\
\hline NuBBE $2435^{2}$ & 356,41 & 3,84 & 5 & 0 & 54,01 & 8 \\
\hline NuBBE $1583^{3}$ & 486,33 & 4,40 & 5 & 2 & 79,29 & 2 \\
\hline SAG $1503[A]^{4}$ & 300,33 & 2,26 & 4 & 1 & 64,68 & 6 \\
\hline $\begin{array}{l}\text { apeso molecular; }{ }^{b} \text { lip } \\
\text { de ligações rotac } \\
\text { skimmiarepin; }{ }^{4} \text { safir }\end{array}$ & $\begin{array}{l}\text { licidade; }{ }^{c} \text { acept } \\
\text { áveis; }{ }^{1}(7 \mathrm{R}, 8 \mathrm{~s} \\
\text { hida. }\end{array}$ & & $\begin{array}{l}\text { loadore } \\
3^{\prime} 4^{\prime}-t e\end{array}$ & $\begin{array}{l}\text { ogênio } \\
\text { oxy-8, }\end{array}$ & $\begin{array}{l}\text { de superfíci } \\
\text { gnan; }{ }^{2} \text { oxo }\end{array}$ & 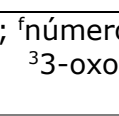 \\
\hline
\end{tabular}




\section{DISCUSSÃO}

A cavidade de ligação do inibidor (IBC, do inglês Inhibitor Binding Cavity) da MAO-B humana é descrita por resíduos como Pro-102, Leu-171, Cys-172, Ile-198, Ile-199, Gln-206, Ile-316, Tyr-326, Phe-343, Tyr-398, Tyr-435 e o anel isoaloxazina de $\mathrm{FAD}^{1,24}$, e os compostos ancorados na IBC interagiram com Tyr-60, Phe-168, Leu-171, Ile-198, Ile199, Gln-206, Tyr-326, Phe-343, Tyr-398, Gly-434 e Tyr435 , indicando uma boa afinidade de ligação. A maior parte da cavidade do sítio ativo é hidrofóbica ${ }^{25}$, o que permite a forte ligação de substratos apolares e inibidores suscetíveis a interações hidrofóbicas e de van der Walls. Entretanto, a única porção hidrofílica está localizada próxima ao anel isoaloxazina de flavina, entre os resíduos Tyr-398 e Tyr-435, que em conjunto formam uma gaiola aromática imprescindível para o reconhecimento e a direcionalidade da funcionalidade amina do substrato ${ }^{17,25}$. Ambos os aminoácidos têm sido utilizados para interações hidrofóbicas pelos NuBBEs 1668 e 1583, enquanto a Tyr-398 foi utilizada para interação hidrogênio pelo 3-oxo-skimmiarepin. Além disso, o Gln-206 interage formando uma ligação de hidrogênio com o ligante de cristalografia, a Safinamida. Entretanto, essa interação não foi reproduzida por nenhum dos três compostos, somente uma interação hidrofóbica ocorreu entre este resíduo e o 3-oxo-skimmiarepin ${ }^{1}$.

O sítio ativo da MAO-B consiste de uma entrada conectada à cavidade do substrato, na qual o resíduo Ile-199 
atua como uma "porta" entre as duas cavidades, e a rotação da cadeia lateral permite a separação ou fusão destas ${ }^{23}$. Inibidores relativamente grandes são capazes de mudar a conformação da enzima de tal forma que estas duas cavidades se fundem formando uma só, exibindo maior afinidade de ligação ${ }^{25}$.

Nossos resultados de interações indicam que os três compostos utilizam ambas as cavidades como potenciais alvos de ligação, tornando-os inibidores potentes da MAO-B, especialmente por dois deles exibiram uma energia de ligação menor que o inibidor de cristalografia Safinamida.

Comparando as distâncias de ligação dos ligantes do NuBBE com as do inibidor, em relação as interações hidrofóbicas a Safinamida só apresentou melhor distância ao interagir com os resíduos de Tyr-398, Leu-171 (quando comparada à mesma ligação com os NuBBE's 1668 e 2435), e com a Ile-199 (somente em comparação com 0 (7R,8S,8'R)-7-Hydroxy-3,4,3',4'-tetramethoxy-8,8'-

neolignan). Contudo, em relação ao resíduo Phe-168, tanto 0 (7R,8S, 8'R)-7-Hydroxy-3,4,3',4'-tetramethoxy-8,8'neolignan quanto o oxovirolin exibiram uma menor distância de ligação que a Safinamida.

Em relação às ligações de hidrogênio, somente 0 (7R,8S,8'R)-7-Hydroxy-3,4,3',4'-tetramethoxy-8,8'-

neolignan conseguiu repetir uma das interações da Safinamida (com a Tyr-60), mas exibindo uma maior distância de ligação. Em ambos os casos, a Tyr apresentouse como doador de ligação de $\mathrm{H}$ e o ligante como aceptor. 
Com exceção das interações n, todas as distâncias de ligação foram iguais ou inferiores a 4,0 $\AA$.

Contudo, a maioria dos compostos candidatos a fármacos falham durante os ensaios clínicos, devido apresentarem um inadequado perfil farmacocinético ${ }^{1}$. Para evitar este acontecimento, as estratégias mais recentes na descoberta e no planejamento de fármacos concentram-se em definir o perfil ADMET de novas moléculas visando a seleção e o desenvolvimento somente de compostos semelhantes a medicamentos que obtenham um perfil farmacocinético ótimo. Para tal, analisamos também as propriedades biológicas dos compostos estudados.

Os bancos de dados NuBBE e PubChem forneceram as propriedades físico-químicas mais relevantes para formação de um perfil farmacocinético dos compostos em estudo ${ }^{18-20}$. Felizmente, nenhum dos compostos estudados violou os critérios de Lipinski e Veber ${ }^{22,23}$ (Tabela 3).

Em relação à classe química, dois dos compostos pertencem à classe dos Lignóides ( $\left(7 R, 8 S, 8^{\prime} R\right)$-7-Hydroxy$3,4,3^{\prime}, 4^{\prime}$-tetramethoxy-8, $8^{\prime}$-neolignan e 2435 ), enquanto 0 3-oxo-skimmiarepin é um terpeno, mais especificamente um triterpenoide (Tabela 1). Nenhum dos compostos apresentou propriedade biológica descrita na literatura, no entanto suas classes químicas já foram estudadas em correlação com a DP. Estudos demonstraram que os terpenoides (ou terpenos) estão presentes em plantas medicinais utilizadas em tratamento de doenças do SNC, incluindo a DP26-28. Outro estudo destaca o extrato vegetal da Aristolochia cymbifera 
(cipó mil homens), comumente conhecida como agente neuroprotetor, devido apresentar em sua composição terpenoides, lignoides e flavonoides ${ }^{29}$.

Além disso, o oxovirolin já foi testado contra leucemia, para análise de atividade antifúngica e atividade antileishmanial, para teste de toxicidade contra macrófagos de camundongos BALB/C avaliados como viabilidade celular. No entanto, o oxovirolin apresentou-se inativo ou inespecífico para os testes de bioensaio ${ }^{19}$.

\section{CONCLUSÕES}

Atualmente, a técnica de triagem virtual tem ocupado papel de destaque entre as estratégias de identificação de novas substâncias bioativas. O presente estudo analisou a estrutura de compostos naturais a fim de explorar novos inibidores de MAO-B, visando o desenvolvimento de fármacos anti-parkinsonianos.

Um total de 382 compostos foram encontrados no banco de moléculas do NuBBE. Após remoção das moléculas duplicadas, permaneceram 174. O filtro CNS revelou que apenas 9 das 174 moléculas apresentaram características físico-químicas necessárias para ultrapassar a barreira hematoencefálica. Foi realizado o docking molecular dessas 9 moléculas junto com o inibidor Safinamida na cadeia A da enzima MAO-B e os dados obtidos demonstram a presença de três inibidores teóricos: (7R,8S, 8'R)-7-Hydroxy-3,4,3',4'tetramethoxy-8,8'-neolignan, Oxovirolin e 3-oxoskimmiarepin. A maioria das interações intermoleculares 
ocorreram entre os compostos e resíduos já comprovados na literatura como presentes no sítio ativo da enzima MAO-B. Destaca-se que os inibidores encontrados apresentam uma afinidade ao sítio de ligação MAO-B melhor ou similar à do inibidor de referência. Além disso, todos os compostos exibiram propriedades físico-químicas favoráveis à biodisponibilidade oral, de acordo com os descritores de Lipinski e Veber.

A comparação por sobreposição de estruturas do complexo cristalográfico com a melhor pose de docking da Safinamida revelou um modo de ligação similar à conformação original com RMSD de 0,76 (Figura 3), estando dentro do valor aceitável como válido (até $1,0 \AA$ ) e validando positivamente o protocolo de docking.

O presente estudo provê informações primordiais para a otimização dos compostos (7R,8S,8'R)-7-Hydroxy3,4,3',4'-tetramethoxy-8,8'-neolignan, Oxovirolin e 3-oxoskimmiarepin atuando como inibidor da MAO-B no tratamento da doença de Parkinson. Entretanto por se tratar de um estudo in silico, é necessário que mais pesquisas e testes sejam feitos para avaliar in vitro e, posteriormente, in vivo o comportamento destas moléculas.

\section{AGRADECIMENTOS}

Agradecimento à Universidade Federal do Oeste do Pará pelo apoio e incentivo a esta pesquisa e ao Laboratório de Pesquisa Labin 02. 


\section{REFERÊNCIAS}

1.Azam F, Mohamed N, Alhussen F. Molecular interaction studies of green tea catechins as multitarget drug candidates for the treatment of Parkinson's disease: computational and structural insights. Network 2015;26:97-115. https://doi.org/10.3109/0954898X.2016.1146416 2.Jayaraj RL, Elangovan N, Dhanalakshmi C, Manivasagam T, Essa MM. CNB-001, a novel pyrazole derivative mitigates motor impairments associated with neurodegeneration via suppression of neuroinflammatory and apoptotic response in experimental Parkinson's disease mice. Chem Biol Interact 2014;220:149-57. https://doi.org/10.1016/j.cbi.2014.06.022

3.Oertel W, Schulz JB. Current and experimental treatments of Parkinson disease: A guide for neuroscientists. J Neurochem 2016;139(Suppl 1):325-37. https://doi.org/10.1111/jnc.13750

4. Lee A, Gilbert RM. Epidemiology of Parkinson Disease. Neurol Clin 2016;34:955-65. https://doi.org/10.1016/j.ncl.2016.06.012

5.PD Med Collaborative Group, Gray R, Ives N, Rick C, Patel S, Gray A, et al. Long-term effectiveness of dopamine agonists and monoamine oxidase B inhibitors compared with levodopa as initial treatment for Parkinson's disease (PD MED): a large, open-label, pragmatic randomised trial. Lancet 2014;384:1196-205. https://doi.org/10.1016/S0140-6736(14)60683-8

6. Poewe W, Seppi K, Tanner CM, Halliday GM, Brundin P, Volkmann J, et al. Parkinson disease. Nat Rev Dis Primers 2017;3:17013. https://doi.org/10.1038/nrdp.2017.13

7.Przedborski S. The two-century journey of Parkinson disease research. Nat Rev Neurosci 2017;18:251-9. https://doi.org/10.1038/nrn.2017.25

8. Lenke TL, Williams DA. Foye's principles of medicinal chemistry. 7th ed. Philadelphia: Lippincott Williams \& Wilkins; 2008, 1479p. http://www.gbv.de/dms/bs/toc/667800042.pdf

9.Carradori S, D'Ascenzio M, Chimenti P, Secci D, Bolasco A. Selective MAO-B inhibitors: a lesson from natural products. Mol Divers 2014;18:219-43. https://doi.org/10.1007/s11030-013-9490-6

10.Nel MS, Petzer A, Petzer JP, Legoabe LJ. 2-Heteroarylidene-1indanone derivatives as inhibitors of monoamine oxidase. Bioorg Chem 2016;69:20-8. https://doi.org/10.1016/j.bioorg.2016.09.004

11.Youdim MB, Edmondson D, Tipton KF. The therapeutic potential of monoamine oxidase inhibitors. Nat Rev Neurosci 2006;7:295-309. https://doi.org/10.1038/nrn1883

12.Robinson SJ, Petzer JP, Petzer A, Bergh JJ, Lourens AC. Selected furanochalcones as inhibitors of monoamine oxidase. Bioorg Med Chem Lett 2013;23:4985-9. https://doi.org/10.1016/j.bmcl.2013.06.050 13. Minders C, Petzer JP, Petzer A, Lourens AC. Monoamine oxidase inhibitory activities of heterocyclic chalcones. Bioorg Med Chem Lett 2015;25:5270-6. https://doi.org/10.1016/j.bmcl.2015.09.049

14. Binda C, Wang J, Pisani L, Caccia C, Carotti A, Salvati P, et al. Structures of human monoamine oxidase $B$ complexes with selective 
noncovalent inhibitors: safinamide and coumarin analogs. J Med Chem 2007;50:5848-52. https://doi.org/10.1021/jm070677y

15.Sander T, Freyss J, von Korff M, Rufener C. DataWarrior: an opensource program for chemistry aware data visualization and analysis. J Chem Inf Model 2015;55:460-73. https://doi.org/10.1021/ci500588j 16.Souza SD. Estudo de inibidores de colinesterases aplicando técnicas de QSAR-2D (HQSAR) e docking molecular (Tese). Rio de Janeiro: Universidade Federal do Rio de Janeiro, 2012. 115 p. http://objdig.ufrj.br/59/teses/793349.pdf

17. Binda C, Newton-Vinson P, Hubálek F, Edmondson DE, Mattevi A. Structure of human monoamine oxidase $B$, a drug target for the treatment of neurological disorders. Nat Struct Mol Biol 2002;9:22-6. https://doi.org/10.1038/nsb732

18.PubChem. Compound summary: (1R,2S,3R)-1,4-Bis(3,4dimethoxyphenyl)-2,3-dimethylbutane-1-ol.

https://pubchem.ncbi.nlm.nih.gov/compound/15386364

19.PubChem. Compound summary: alpha-[2-Methoxy-4-[(E)-1propenyl]phenoxy]-3',4'-dimethoxypropiophenone.

https://pubchem.ncbi.nlm.nih.gov/compound/6279288

20.PubChem. Compound summary: 3-Oxoskimmiarepin. https://pubchem.ncbi.nlm.nih.gov/compound/101671907

21. Maiorov VN, Crippen GM. Significance of root-mean-square deviation in comparing three-dimensional structures of globular proteins. J Mol Biol 1994;235:625-34.

https://doi.org/10.1006/jmbi.1994.1017

22.Lipinski CA. Drug-like properties and the causes of poor solubility and poor permeability. J Pharmacol Toxicol Methods 2000;44:235-49. https://doi.org/10.1016/s1056-8719(00)00107-6

23.Veber DF, Johnson SR, Cheng HY, Smith BR, Ward KW, Kopple KD. Molecular properties that influence the oral bioavailability of drug candidates. J Med Chem 2002;45:2615-23. https://doi.org/10.1021/jm020017n

24.De Colibus L, Li M, Binda C, Lustig A, Edmondson DE, Mattevi A. Three-dimensional structure of human monoamine oxidase $A$ (MAO A): relation to the structures of rat MAO $A$ and human MAO $B$. Proc Natl Acad Sci USA 2005;102:12684-9. https://doi.org/10.1073/pnas.0505975102

25.Binda C, Li M, Hubalek F, Restelli N, Edmondson DE, Mattevi A. Insights into the mode of inhibition of human mitochondrial monoamine oxidase $B$ from high-resolution crystal structures. Proc Natl Acad Sci USA 2003;100:9750-5. https://doi.org/10.1073/pnas.1633804100

26. Andrade IGS. Benefícios e Riscos das Plantas Medicinais na Doença de Parkinson (Dissertação). Coimbra: Faculdade de Farmácia, Universidade de Coimbra, $2018.2 \mathrm{p}$. https://estudogeral.uc.pt/bitstream/10316/84376/1/DOCUMENTO\%2 oUNICOpdf.pdf 
27.Rai SN, Birla H, Zahra W, Singh SS, Singh SP. Immunomodulation of Parkinson's disease using Mucuna pruriens (Mp). J Chem Neuroanat 2017;85:27-35. https://doi.org/10.1016/j.jchemneu.2017.06.005 28.Santos DA. Avaliação das possíveis propriedades neuroprotetoras do extrato metanólico de Bauhinia microstachya Raddi e da mistura ae B-amirina sobre o sistema nervoso central de roedores com a doença de Alzheimer e a doença de Parkinson induzidas quimiicamente (Dissertação). Itajaí: Universidade do Vale do Itajaí, 2013. 117p. http://siaibib01.univali.br/pdf/Diogo\%20Adolfo\%20dos\%20Santos.pd f

29. Melo HB. Atividade neuroprotetora do extrato etanólico de Aristolochia cymbifera sobre o sistema nervoso central e periférico de vertebrados (Trabalho de Conclusão de Curso). São Gabriel: Universidade Federal do Pampa, 2016, 42p. http://dspace.unipampa.edu.br/bitstream/riu/1332/1/Atividade\%20n europrotetora $\% 20$ do $\% 20$ extrato $\% 20$ etan $\%$ c3\%b3lico\%20de\%20arist olochia $\% 20$ cymbifera $\% 20$ sobre $\% 200 \% 20$ sistema\%20nervoso\%20ce ntral\%20e\%20perif\%c3\%a9rico\%20de\%20vertebrados.pdf 